\title{
Hate speech in intercultural encounters
}

Guest editors:

Dr. Anna Birgitta Nielsen, Oslo Metropolitan University

Dr. Ekaterine Pirtskhalava, Faculty of Psychology and Education Sciences, Ivane Javakhishvili Tbilisi State University

Ekaterine Basilaia, Faculty of Social and Political Sciences, Ivane Javakhishvili Tbilisi State University 
The issue of hate speech has been a topic of international debate-most frequently in the domains of law, philosophy and language. Different issues linked to changes in society, ranging from the proliferation of social media, innovation and technology and influx of fake news, disinformation and propaganda to the rise of nationalism, far-right movements, increased cross-border movement of people and transnational business have made studying the conceptual and practical aspects of hate speech in different contexts ever more important.

The papers in this issue focus on the sociolinguistic aspects of the use of hate speech and its different variants in online communication, offering a much-needed perspective on how hate speech in digital communication can be identified and tackled. For example, Dr. Manana Ruseishvili and Dr. Rusudan Dolidze analyse hate speech in computer-meditated communication, focusing on the polylogal, asynchronic remarks made by members of the public reacting to articles in online media or press releases about the LGBT pride event planned for June 2019. The research carried out by Lilit Bekaryan explores how hateful posts and comments can start among Facebook users, and studies the language means employed in their design based on data from more than ten open Facebook pages managed by popular Armenian figures, such as media experts, journalists, politicians and bloggers. Dr. Tatjana R. Felberg explores the interconnectedness between impoliteness and hate speech in online comments in Croatia and Serbia by applying impoliteness theory and a critical discourse analysis framework. Her research demonstrates that those who post often fluctuate between hate speech and impoliteness. Dr. Ayunts and Dr. Paronyan provide a comparative analysis of manifestations of hate speech and euphemisms in Armenian and British online media outlets and social sites targeting people's sexual orientation with emphasis on the interconnectedness of hate speech and culture.

This issue was prepared as part of the project 'Intercultural encounters in academia and work places in South Caucasus and Norway' funded by DIKU, the Norwegian Agency for International Cooperation and Quality Enhancement in Higher Education. The project partner universities are Oslo Metropolitan University, Ivane Javakhishvili Tbilisi State University, Yerevan State University and Khazar University. 